\title{
Imaging Physical Properties and Dynamics of Dendritic Spines by Atomic Force Microscopy
}

\author{
B.A. Smith,* P.H. Grütter,* and Y. De Koninck** \\ * Physics Dept., McGill University, 3600 University St., Montréal, Québec H3A 2T8, Canada \\ ** Neurobiologie Cellulaire, Centre de recherche Université Laval Robert-Giffard, 2601 chemin de \\ la Canardière, Beauport, Québec G1J 2G3, Canada
}

The formation and stabilization of neuronal synapses is a topic that has attracted much attention in recent years. Dendritic spines are submicron-sized, actin-rich protrusions from dendrites, which form the postsynaptic region of synaptic contacts [1]. Dendritic spines thus form a key element in neuronal communication. Recent studies have shown rapid formation and remodeling of dendritic spines, during development and following intense synaptic activity [2,3]. These findings call for the ability to study, in real time, the ultrastructural properties of spines, their dynamics and physical properties such as elasticity in live cells.

Traditionally, dendritic spines have been studied with transmission electron microscopy (TEM) and optical techniques such as confocal microscopy. TEM has provided high-resolution images of spines, revealing the distribution of internal actin filaments and other structures. However, the ability to study dynamic events with this technique is hampered by its inapplicability for studies of live tissue. Confocal microscopes lack the ability to resolve internal structures of spines, but can be readily applied to acquire time-lapse sequential snapshots of fluorescence-labeled spines in living neurons $[1,3]$. The resolution of this type of imaging is, however, fundamentally limited by optical diffraction.

In recent years, the atomic force microscope (AFM) has emerged as a versatile tool for imaging biological specimens with nanometer scale resolution [4]. The AFM can image topological features of bio-surfaces in physiological environments, and can also be used to simultaneously probe localized physical properties such as elasticity [5] or adhesion [6]. Recent technological advances have made it possible to non-destructively image soft tissue on time scales that are relevant to study dynamic events and growth of filopodial protrusions from dendrites, which lead to the formation of spines.

In this work we combine the techniques of confocal and atomic force microscopy to study spine dynamics and elasticity in live hippocampal cultures. AFM elasticity mapping reveals regions within the spines that are stiffer than the surrounding tissue. These regions likely have a high density of actin filaments that produce the mechanical drive for spine growth. The rapid remodeling of these protrusions are imaged with both confocal and AFM. The resolutions, both spatial and temporal, are compared for the two techniques. This type of AFM work contributes significantly to the study of neuronal dendritic spines in both its resolution and its ability to provide measures of elastic properties.

References

[1] A. Matus Science 290 (2000) 754.

[2] M. Maletic-Savatic et al. Science 283 (1999) 1923. 
[3] G.S. Marrs et al., Nat. Neurosci. 4 (2001) 1006.

[4] D.J. Mueller et al., Biochim Biophys Acta 1460 (2000) 27.

[5] C. Rotsch and M. Radmacher, Biophys J. 78 (2000) 520.

[6] M. Grandbois et al. J Histochem Cytochem. 48 (2000) 719.
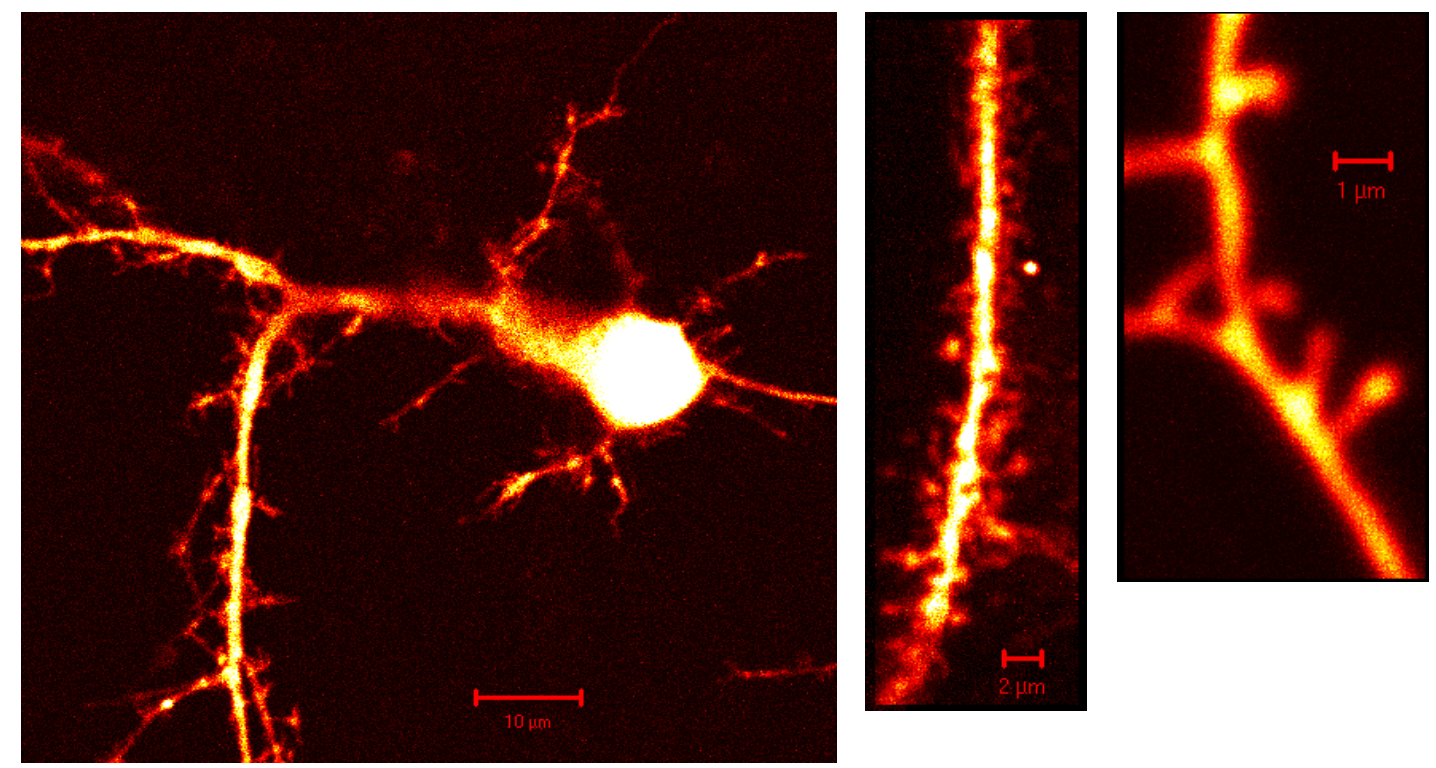

FIG. 1. Confocal images of a cultured hippocampal neuron (left) transfected with Green Fluorescent Protein (GFP). Note the profuse distribution of spines protruding from the dendrite (middle). However, even at high power (right) the details of the spine morphology are poorly resolvable.
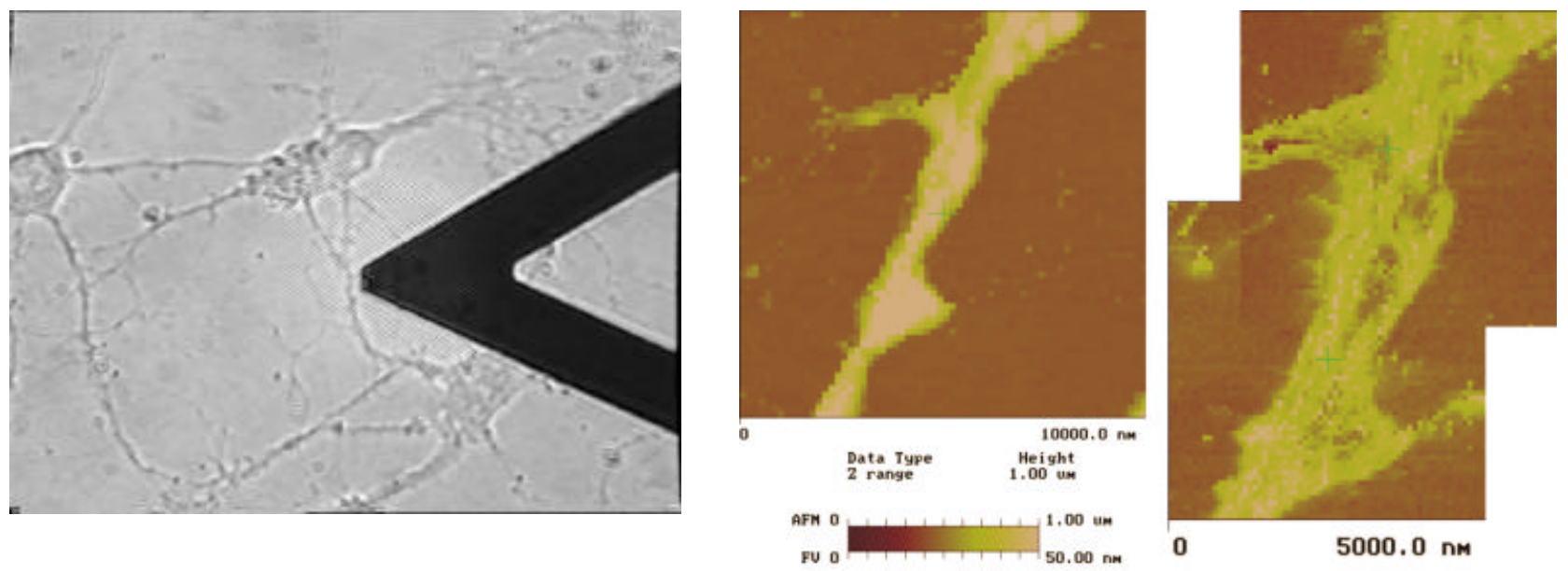

FIG. 2. Photomicrograph of the AFM probe during imaging of a dendrite in a culture of hippocampal neurons (left). The AFM images were acquired in force-volume mode. The topographic image (middle) shows a dendrite with a few protrusions. The elasticity contrast map (right) allows one to locate stiff regions within the dendritic shaft and the developing spines. These regions are likely rich in actin filaments. 